\title{
Vivre ensemble ou la douleur d'être « en grande famille »1
}

\author{
«Le pardon n'implique pas l'oubli » \\ «La mort n'est pas une nécessité, c'est une obligation »
}

C.

\begin{abstract}
Résumé : La famille africaine a souvent été magnifiée pour sa convivialité consensuelle et sa solidarité organique apparente. En réalité, une expérience plus intime des rapports individuels au sein du couple ou entre frères et sœurs révèle la violence des conflits qui l'habitent et la part d'arbitraire des régulations qui la fondent. Face à l'enchaînement des menaces directes ou voilées, le membre d'une " grande famille " dispose en fin de compte, à la différence des individus des sociétés post-industrielles, d'un faible éventail de recours possibles.
\end{abstract}

Summary : The African family has often been magnified by its consensus of conviviality and its apparent organic solidarity. In fact, the intimate experiences of individual relations at the heart of a couple or between siblings reveals the violence inherent in the conflicts they live and the effects of arbitrary regulations which the family is builted on. In the end, in the face of sequences of direct or indirect threats, a member of an extended family has a restricted number of possible appeals, contrary to individuals living in post-industrial societies.

Si l'atelier ou la rue sont autant de «carrefours » ordinaires de la violence visible, la concession familiale, - qu'il s'agisse de cours rurales ou de carrés citadins -, souvent marquée par la promiscuité, l'imbrication des parentèles et les difficultés économiques, sur fond d'histoires de vie faites de ruptures, jalousies et de ressentiment, peut constituer un autre « espace social » où s'exprime, une violence ${ }^{2}$, tantôt larvée, tantôt explicite, souvent banalisée.

En effet, pour des individus dont la satisfaction des besoins essentiels - manger, se loger, se soigner, se déplacer - est chaque jour une conquête renouvelée, l'espace familial n'assure pas nécessairement un rôle protecteur et médiateur, ni par rapport à l'environnement extérieur (protection physique et sécurisation économique), ni même pour ses membres dans leurs interrelations (régulation des conflits). En effet, contre toute attente, les manifestations de la violence restent souvent imparfaitement régulées et rarement désamorcées, et sont même parfois avivées par la nature même des rapports interindividuels, fortement marqués par les différences de statut et de pouvoir et par les disparités des conditions de vie.

\footnotetext{
${ }^{1}$ Ce terme désigne une unité résidentielle constituée d'un ou plusieurs ménages polygamiques auquel vient s'ajouter des collatéraux (enfants placés par exemple) et des employés domestiques.

${ }^{2}$ Par violence familiale, nous considérons toute action verbale, physique ou psychologique, imparfaitement régulée, qui transforme un rapport hiérarchique ou une relation plus égalitaire vers une plus grande domination et soumission.
} 
De ce fait, la cour familiale semble à la fois constituer un réceptacle des formes de violence extérieure et un point d'observation privilégiée des tensions violentes qui mettent à l'épreuve la cellule familiale. L'éventail de ces formes de domination et de confrontation est large : il va de la décision imposée en terme de prise de parole, de mariage ou de déplacement, à la dépossession arbitraire d'un bien et de son usufruit en passant par la menace et l'exclusion. C'est pourquoi sous des dehors de solidarité, d'esprit communautaire, d'hospitalité, de propension à jouir du présent, du sens de la fête, la famille africaine mêle dans l'indicible des sentiments aussi contrastés que le respect et la crainte, l'affection et la distance, l'amour et la haine, la convivialité et la solitude. Par ailleurs, la précarité renforcée des conditions d'existence, après plus d'une décennie de crise et d'ajustement structurel, n'est pas de nature à favoriser des rapports interindividuels fondés sur la confiance et le dialogue. Qui plus est, la médiation de l'affect et de l'intimité parfois faite par la famille constitue une douloureuse mise à l'épreuve du lien social et des sentiments. En réalité, pour tous les protagonistes interrogés, la douleur d'être ensemble ne s'enracine pas seulement dans les manifestations ouvertes de jalousies, dans les déclinaisons protéiformes du soupçon et de la menace, ni même dans les accusations et les coups portés. Ce qui donne à la violence « en grande famille » une intensité tragique peut-être plus grande que dans nos sociétés post-modernes, c'est d'abord cette difficulté fondamentale de l'individu à faire évoluer la nature des liens qui le lie à sa propre famille, voire à s'en distancier; mais c'est aussi l'ambivalence extrême des comportements des membres qui la composent, y compris les plus proches (mari, et père) avec lesquels l'individu est « condamné » à vivre.

Ce texte s'inspire essentiellement de l'histoire récente des rapports interpersonnels au sein de deux concessions polygamiques - en milieu rural sahélien burkinabé (ethnie gourmantché) et en milieu urbain béninois (ethnie goun*), en privilégiant le point de vue des victimes. Nous avons donné à ce dernier exemple une place plus grande, compte tenu de notre intimité partagée sans pour autant négliger les formes plus banales ou plus feutrées observées en milieu rural.

Notre propos n'est pas de fournir un modèle conceptuel englobant, hiérarchisant les facteurs de risque de violence, pondérant les déterminants dans une parfaite représentativité $^{3}$ afin de proposer des itinéraires de médiation. Les violences observées au sein de la "grande famille » renvoient moins à une insécurité civile envahissante ou à une "gender and physically-based violence ${ }^{4}$ qu'à un mode d'expression du sujet en lutte. Ici l'imbrication des segments de parenté, les formes réelles ou supposées de réussite - même transitoires, les différences de statut et les luttes du sujet en individuation, vis-à-vis des autres comme de lui-même, constituent un terreau propice à l'émergence des violences ordinaires. Procès d'intention, mises en accusation, tentatives de fuite et refus n'y sont, en fin de compte, pas très différents de ceux qui occupent la scène publique. Ils doivent être compris, non seulement, comme la pérennisation d'un mode de régulation communautaire mais également comme des éléments avancés d'une quête de pouvoir au sein du groupe. Toutefois, c'est bien par la mobilisation des croyances et craintes (dans la religion révélée ou traditionnelle africaine) que la violence familiale trouve son achèvement tragique.

A travers le thème de la violence domestique, c'est la nature même du rapport à l'autre qui est en question : comment la communauté familiale (comme microcosme au sein de la société), produisant autant de «social », peut-elle générer autant de violence normée ou anomique tout en la banalisant, si ce n'est parce que la violence est une forme d'intensification concurrentielle des relations sociales ? Quel type de lien familial peut

\footnotetext{
* L'ensemble des termes vernaculaires sont en goun.

${ }^{3}$ S. Salzinger et al., "An ecological framework for understanding risk for exposure to community violence ad the effects of exposure on children and adolescents", New York, Elsevier Science, Aggression and Violence Behavior, 2002, 7, 423-451.

${ }^{4}$ R. Jewkes et al., "Risk factors for domestic violence : findings from a South African cross-sectional studdy”, New York, Elsevier Science, Social Science and Medicine, 2002, 55, 1603-1617.
} 
perdurer après des enchaînements et déchaînements réitérés de violence ? L'individu dominé dans sa «cour » peut-il devenir citoyen dans sa propre famille?

\section{Sous le regard des autres}

Pénétrer dans l'intimité familiale, c'est se replonger dans la problématique du lien sous contrainte, de la solidarité redistributive, de l'utilitarisme partagé et de la dette ${ }^{5}$. Car être en famille, c'est avoir l'assurance de n'être jamais « isolé ", de pouvoir bénéficier de soutiens en cas de besoin dans des sociétés où les incertitudes sont récurrentes et multiples. Mais c'est aussi - comme le révèlent la plupart des histoires de vie - vivre sous le regard des autres, parfois bienveillant mais souvent pesant ou même coercitif puisque le comportement de chacun est réexaminé à l'aune d'une «tradition», sans cesse réinventée, parfois magnifiée, qui circonscrit étroitement la marge de manœuvre dont le sujet dispose. En ville comme au village, " l'individu est rarement perçu comme une personne, un sujet autonome et responsable. Il est davantage un élément avancé dans la stratégie du groupe familial ou lignager ${ }^{6}$. Chacun a une place établie en vertu d'un dispositif hiérarchique, fondé sur des différences d'âge, de sexe, de richesse, et sur l'historicité des rapports internes de parenté qui fixent durablement les rapports d'autorité qui peuvent parfois évoluer vers des rapports de force. En cas de contestation, " grands frères », «pères » et «tontons » s'efforceront de pérenniser, par des conseils et, si besoin, par le chantage et la menace les formes héritées de contrôle sur les plus jeunes, les déviants ou les plus vulnérables. De toute évidence, l'autorité s'exerce préférentiellement à l'encontre de ce type d'individu. Tout se passe comme si le groupe entendait maintenir ou reconstituer, coûte que coûte, au besoin par la contrainte, une forme d'entente consensuelle ( $f i f a^{*}$ ) ou de concorde communautaire quand bien même la confiance interindividuelle (aymisséa*) serait inexistante.

Aux situations de violence familiale explicite - verbale (gbeyannamè*) ou physique $\left(g b o h i h o m i^{*}\right)$, nous avons associé les manifestations moins spectaculaires qui travaillent l'individu au quotidien, prenant appui sur des relations d'autorité, et qui disparaissent souvent comme « digérées » par la communauté d'origine qui perçoit parfois mal leur dégradation progressive.

En 1985, C. dont le commerce de tissus «marchait fort » a remis l'équivalent de 6 millions de francs CFA en naïras ${ }^{7}$ à un oncle paternel établi au Nigeria pour effectuer une banale opération de change. Mais ce dernier « grand quelqu'un » à Djofi (pays yorouba) a préféré réinvestir cette somme pour moitié dans ses propres activités et pour moitié dans des prêts d'argent. La dévaluation brutale du naïra est venue ruiner son commerce annulant toute espérance de remboursement. A cette annonce, $C$. a pris la route pour se rendre chez l'oncle indélicat. En pleine nuit, elle est venue lui réclamer son argent, "que j'ai cherché dans la chaleur, dans la fraîcheur, dans la pluie et la fatigue ». S'est ensuivie une discussion hachée et houleuse. "Si tu ne me rembourses pas, mes aïeux viendront te réclamer çà ; Dieu viendra tout prendre chez toi ». "Tu ne peux rien contre moi » a-t-il rétorqué. De retour à Cotonou, C. a d'abord voulu déposer une plainte pour escroquerie au Tribunal. Ce que son époux lui a vivement déconseillé parce qu'elle risquait d'être «tuée » en sorcellerie. Certes, deux ans plus tard en 1987, à l'occasion du décès de la grand-mère paternelle de $C$., la dette de l'oncle a trouvé une compensation symbolique dans le fait que ce dernier a participé aux funérailles organisées par la «grande famille », avec le don d'un bœuf et d'un mouton, mais pour Colette il n'en reste pas moins que la dette réelle court encore. Ce

\footnotetext{
${ }^{5}$ A. Marie, «Individualization Strategies Among City Dwellers in Contemporary Africa : Balancing the Shortcomings of Community Solidarity and the Individualism of the Struggle for Survival », International Review of Social History, 2000, 45, 137-157.

${ }^{6}$ P. Janin, L'avenir des planteurs camerounais, Paris, Karthala, 1999, p. 198.

${ }^{7}$ L'essentiel des ses ventes étaient, en effet, réalisées à cette époque avec des intermédiaires nigérians.
} 
cas montre bien comment, à l'aune du complexe hiérarchique, peuvent se nouer des décisions collectives qui marginalisent et humilient les plus faibles : l'oncle débiteur peut ainsi violenter sa nièce, le mari menacer son épouse et lui intimer l'ordre de se taire.

En milieu rural burkinabé, le pouvoir discrétionnaire de la famille élargie n'est pas moins fort. P. née à Thion en 1949, une petite préfecture rurale de l'Est du Burkina, en a ainsi fait la douloureuse expérience. Mariée en 1964, elle a émigré à Mani, distante d'environ 30 kilomètres, dans une grande cour polygamique (qui regroupait 15 ménages, soit environ 150 personnes en 2001). Dans cette zone agropastorale sahélienne enclavée, les conditions de vie restent éminemment précaires et aléatoires, rythmées par le caractère saisonnier des activités et la récurrence des périodes de soudure ${ }^{8}$ alimentaire sur fond de raréfaction progressive des ressources en eau, en bois et en terre cultivable. En 1991, à la mort de son mari, sa situation se dégrade brutalement. En butte à la pression familiale, elle doit quitter la « grande cour » et rejoindre un hameau de culture, distant d'environ trois kilomètres, à l'écart du village et où son mari avait l'habitude de résider en période des travaux agricoles (de juin à octobre). Et les frères du défunt de profiter du départ de la veuve pour récupérer certaines parcelles de village possédées par son mari, car elles avaient de meilleures potentialités agricoles, de même que les cases d'habitation et le petit bétail. En 1996, toutefois un des frères du défunt lui attribuera un droit de culture temporaire sur une parcelle de brousse, hors du village. Sans charrue, ni animal de trait, là voilà désormais seule avec sa fille (âgée de 35 ans) et un garçon plus jeune (15 ans) pour cultiver deux petites parcelles de sorgho et d'arachide. Les relations avec les autres ménages de la cour se sont progressivement un peu plus distendues tandis que les promesses d'aide se raréfiaient. En situation précaire, elle vit au jour le jour sans pouvoir faire face aux imprévus. Ainsi, faute de pouvoir acheter des céréales au marché (ses deux derniers moutons ont été vendus au cours de la soudure précédente), elle a dû procéder à des ajustements drastiques pour survivre entre avril et octobre 2002 : consommer des feuilles de cueillette plus que de coutume et réduire sa ration céréalière des deux-tiers, mettre en gage sa récolte d'arachides pour acheter 15 yorouba ${ }^{9}$ de maïs. Il est difficile de ne pas voir dans la marginalité imposée par le groupe familial, dans l'absence d'aide et de recours - alors même que son époux participait activement à cette économie morale de la redistribution - une forme de violence enracinée dans la régulation sociétale, pour laquelle la solidarité se fonde davantage sur des liens organiques (solvabilité, espérance de gain en retour) que mécaniques.

L'exemple qui suit illustre mieux encore comment normes sociales intériorisées et précarité économique s'enchevêtrent pour engendrer un «ordre » familial qui conduit à sécuriser le groupe contre l'individu et à privilégier (dans ce cas précis) le long terme sur le temps immédiat comme ordre banal des choses, le tout dans la méconnaissance de l'arbitraire qui fonde cet ordre. Il a pour cadre une concession rurale d'immigrés mossi, située dans la zone sahélienne orientale du Burkina Faso. Février 2002. Sept heures du matin. Installé sur une « chaise-fauteuil », placée par le chef de ménage à l'entrée de la concession, je suis adossé à une case ronde en banco. D'ici, je peux embrasser l'ensemble de l'espace familial. En face de moi, attaché à un poteau, un bœuf d'embouche rumine, sous un abri en secco. C'est le bœuf du chef de ménage, S., trente ans, "rapatrié ${ }^{10} \mathrm{de}$ Côte d'Ivoire en janvier 2001. Lavé régulièrement, nourri chaque jour de tiges de mil et abreuvé d'eau blanche (un mélange d'eau et de céréales écrasées) par son épouse et ses enfants, ce bœuf représente la principale ressource du ménage une fois épuisés les

\footnotetext{
${ }^{8}$ La soudure correspond à une période de déficit alimentaire de durée et d'intensité variable que l'on peut estimer en nombre de jours (entre la fin d'utilisation des greniers et la prochaine récolte céréalière) ou en nombre de sacs (rapporté aux besoins totaux théoriques du ménage).

9 Il s'agit de l'unité de mesure, valant environ $3,5 \mathrm{~kg}$ de céréales, la plus communément utilisée par les achats et la préparation céréalière.

${ }^{10}$ Ce terme désigne les émigrants burkinabé rentrés précipitamment «au pays » depuis le début des troubles politiques ivoiriens.
} 
greniers céréaliers. Ce matin, son propriétaire espère d'ailleurs la visite d'un acheteur potentiel, un rabatteur qui parcourt la brousse pour son patron, commerçant de bétail à Pouytenga, un grand marché de la zone. Il pense pouvoir négocier une vente autour de 200.000 francs CFA, ce qui paraît élevé étant donné l'offre surabondante en cette période de l'année. Puis, brusquement, et sans raison apparente, il apostrophe violemment les membres de la cour. Son ire semble être motivée par le retard des soins habituellement prodigués. Les heures passeront dans l'attente de la visite du rabatteur sans que personne, pas même les enfants en bas-âge, ne mange. Quant à moi, il me sera proposé une calebasse d'arachides grillées et un bol de tô que je ne pourrai refuser. Je mangerai néanmoins à contrecœur, sous le regard fixe des enfants, immobiles et muets.

Le discours que tiennent les «petits", les faibles ou les « opprimés » tend également à accréditer l'idée d'une violence si commune et si proche qu'elle en est banalisée et occultée. Ainsi, le premier entretien approfondi réalisé en décembre 2002 à Mani avec Pourga, une veuve chef de ménage gourmantché, fut laconique. Elle avait une nette propension à embellir la nature de ses relations avec les frères du défunt, insistait sur la bonne entente avec son voisinage, tout en déplorant l'absence du défunt. Or, sans transition, elle révéla qu'elle était dans l'impossibilité de se défendre contre toute décision collective ou abus, qu'elle connaissait une sous-alimentation récurrente. Elle confirmait, de manière presque caricaturale, l'existence d'une violence sociale « discrète ", intégrée et acceptée au même titre que la faim, la maladie et la mort brutale. A Cotonou, dans le cas de C., une épouse chassée par son mari, la situation est quelque peu différente. Quelque peu théâtralisée au cours de deux importantes réunions familiales très tendues en 1991 et 1992, la violence verbale et psychologique (menaces sorcières principalement) de son mari à laquelle participe activement une partie de la famille a été très douloureusement ressentie de même que les tentatives de résolutions arbitraires du conflit. Dans un premier temps, loin de réfuter les décisions éventuelles du conseil de famille, dont elle pressent néanmoins le caractère partial en raison de relations privilégiées de son époux avec les autres figures masculines, elle s'y soumet par anticipation. C'est, soutenue par une foi inextinguible en son « innocence » et en « son » Dieu que cette épouse et mère s'est longuement exprimée sur les accusations de sorcellerie dont elle faisait l'objet de la part d'une partie du ménage polygamique.

"Cela fait vingt-sept ans que vous connaissez mon père (que nos familles sont liées par mon mariage avec B.). Je ne suis jamais venue vous voir pour vous dire que je souffre. J'ai tout supporté pour mes enfants (4 fils et 2 filles). Je ne sais pas si quand on est malade c'est un être humain qui fait cela (si maladie et échec peuvent désirés et produits par une personne). Je sais que vous (les parents de la deuxième épouse) allez tout faire contre moi. Dites à la famille de B. de tout faire contre moi. Je sais que je n'ai rien fait. J'accepte tout (toute vengeance, punition et infamie) ».

\section{La part du diable ou la jalousie sans fin}

Dans la famille, la violence se manifeste aussi souvent de manière indirecte ${ }^{11}$. Elle est alors soit détournée vers le monde de l'invisible pour nourrir des luttes répétées $\left(a z e^{*}\right)$, soit canalisée au profit de représentations théâtralisées de la confrontation (les conseils de famille). Loin d'affaiblir les structures de contrôle et de régulation, ces détours les confortent par la recherche systématique de boucs émissaires. Elle renvoie donc paradoxalement à un mode de régulation qu'elle est censée dénoncer et affaiblir. Qui plus est, cette forme de violence se maintient en permanence dans les esprits sans jamais trouver de terme définitif. Elle vient donc hanter les rapports sociaux visibles les plus ordinaires entre proches comme son envers menaçant.

${ }^{11}$ P. Geschiere, Sorcellerie et politique en Afrique. La viande des autres, Paris, Karthala, 1995, p. 32. 
Arme des faibles, revanche des aigris, la sorcellerie vient surtout renforcer le pouvoir des plus « grands". Elle est bien à la fois « une ressource ou un capital d'ordonnancement ${ }^{12}$ et un moyen d'action sociale ${ }^{13}$. Paradoxalement ceux mêmes qui la mettent en avant sont aussi ceux qui la craignent le plus. Parfois au contraire, dans le cas que nous décrirons, l'accusation de sorcellerie est explicitement formulée et ne sert pas à dissimuler des acteurs individuels ou collectifs.

Ainsi, elle chemine durant trois décennies au cœur d'un ménage polygamique de Cotonou mais n'opère réellement à découvert qu'à partir des années 1990 à l'occasion de deux violentes confrontations familiales. Nourrie, dès l'origine par un sentiment banal de jalousie (ahouanto*), tant affective, religieuse qu'économique, entre deux co-épouses, cette violence sera au fil des années progressivement relayée par certains membres du ménage, accréditant la thèse du « complot» (yéblassé*) lignager collectif. Par sa durée, son intensité dramatique, la diversité des formes d'opposition violente et les conséquences à moyen terme sur le devenir des enfants, cette histoire à épisodes constitue un véritable tableau synoptique de la violence familiale ordinaire.

B., comptable de profession, est né à Porto-Novo (pays goun) en 1933. Il a épousé coutumièrement en 1955, R., née vers 1935 dans la région d’Abomey (province du Zou). De leur union, naîtront sept enfants entre 1955 et 1973 (5 fils et 1 fille). Très vite, la grande famille a reproché à $R$. (la première épouse) de ne pas bien entretenir ses enfants et de mal s'occuper de son mari. Au point d'imposer une épouse de village (la deuxième) à B. en 1963 avec laquelle il aura trois enfants. En 1965, il fait la rencontre de C., âgée de 17 ans, née également à Porto-Novo. Cette dernière travaillait à l'époque comme fille de salle à Cotonou dans la clinique de son tuteur qui était un ami de B. Elle tenait parallèlement un petit commerce de tissus et de chaussures. L'année suivante, $B$. et $C$. (la troisième épouse) se marient civilement à la Mairie de Cotonou. Entre 1967 et 1979, C. aura 6 enfants avec B. (4 garçons et 2 filles). Jusqu'en 1972, la cohabitation entre ces deux épouses était relativement paisible, chacune occupant une cour, louée par B. Cette année-là, un changement d'importance interviendra. En effet, B. - après avoir acquis pour 250.000 francs une parcelle dans le quartier d'A. en 1968 - entreprend la construction d'une maison imposante à deux étages. Dès leur entrée respective dans cette cour, la mésentente s'installa. "Je n'ai pas été accueillie comme la petite femme. Du tout...» se rappelle C. aujourd'hui. Bien au contraire, dès le début R. a annoncé qu'elle souhaitait rester seule dans cette cour. Menaces, bagarres se sont alors succédées. «Tu seras la première à partir » rétorquait Colette quant Régina la menaçait d'un couteau ou de la mordre sans que sa plaie ne puisse guérir. A cette époque, «le tour de préparation » de la cuisine pour l'ensemble des membres du ménage durait un mois. Néanmoins, B. manifestait une préférence marquée pour la cuisine de $C$. et semblait apprécier la complicité affectueuse qui les réunissait. Il avait, en outre, coutume de la complimenter pour les soins attentifs apportés aux enfants (tout particulièrement dans le domaine de l'habillement et de l'hygiène).

A partir de 1982, à cette jalousie affective est venue s'ajouter une forme de rivalité économique. Installée à Lomé depuis 1979 (tandis que R., la grande épouse, était restée à Cotonou) avec son époux qui avait trouvé un emploi de comptable dans une société d'import-export, C. revenait souvent à Cotonou où ses activités commerciales «marchaient fort »: elle possédait une boutique en dur au marché de $D$. et vendait des tissus jusqu'au Nigeria. Ce commerce pouvait générer des bénéfices importants, de l'ordre de 500.000 francs CFA mensuels en moyenne et de $\mathbf{1 . 0 0 0 . 0 0 0 ~ d e ~ f r a n c s ~ C F A ~ e n ~ d e ́ c e m b r e ~ a ̀ ~}$ l'occasion des fêtes. Ses bénéfices étaient immédiatement réinvestis dans son fonds de commerce. De ce fait, elle pouvait activement contribuer à la vie du ménage en fournissant l'argent de la popote et en achetant des vêtements pour ses enfants. Or, de

\footnotetext{
${ }^{12}$ F. Bernault et J. Tonda, «Dynamiques de l'invisible en Afrique », Paris, Karthala, Politique Africaine, 2000, $\mathrm{n}^{\circ} 79$, pp. 5-16.

13 B. Jewsiewicki, Naître et mourir au Zaïre. Un demi-siècle d'histoire au quotidien, Paris, Karthala, Collection « Les Afriques », 1993, 255 p.
} 
son côté, R. n'exerçait aucune activité d'envergure en dehors d'une petite vente à emporter et montrait un penchant certain pour la boisson. La comparaison était rude, pour ne pas dire humiliante. C'est pourquoi, R. a longtemps voulu croire que la réussite outra-geuse de $C$. dépendait uniquement de l'aide financière de leur époux. Il n'en était rien sauf pour un camion acheté 4.500.000 francs CFA pour moitié, moitié en 1985.

L'année 1986 marque un tournant décisif pour C. En septembre, l'Etat nigérian dévalue de $70 \%$ le naïra pour faire face à une grave crise financière. Du jour au lendemain, le rouleau de tissu acheté plus 70.000 francs CFA, revendu 200 naïras à des grossistes nigérians, n'en valait plus que 22.000 francs CFA. Son commerce de riz importé, qui atteignait un volume de 2.000 sacs par trimestre « tombait » également brutalement. Les acheteurs nigérians s'étaient immédiatement détournés de Cotonou, préférant importer directement du riz asiatique. Un reliquat de 1.200 sacs de $50 \mathrm{~kg}$, achetés 9.500 francs CFA l'unité, qui commençaient à moisir avait du être bradé à 7.000 francs CFA. «R. était contente de ma chute » commente tristement $C$. qui ne se relèvera jamais vraiment de cette faillite.

En 1989, R. opère un revirement confessionnel ; elle délaisse la religion vodoun traditionnelle et $s$ 'entiche de la religion catholique que pratiquait $B$. et $C$. Elle passe sa première communion en 1990 et exprime le souhait de faire reconnaître religieusement son union avec B. Cela s'avère impossible étant donné la situation de polygamie avérée. D'où une volonté pugnace de rester la seule et unique épousée. Cette piété nouvelle résultait-elle d'un véritable acte de foi ou n'était-elle qu'un stratagème dans une compétition qui lui était défavorable ? A partir de 1991, la crise qui opposait les deux femmes prend une autre dimension. Elle va progressivement englober toute la famille élargie, mêlant manipulation religieuse, menace sorcière, subornation de témoin pour culminer par l'ex-pulsion (1993) et le divorce de C., la petite femme (2001).

Nous tenterons de relater ici le plus fidèle possible l'enchaînement des événements dramatiques au cours de la décennie 1990 en dépit de leur complexité et d'une certaine confusion. En 1990-1991, E. la fille aînée de R. était en année de BTS secrétariat à Abidjan. Elle semblait bien réussir jusqu'à ce qu'elle contracte une maladie nerveuse au cours de l'été 1991 et se retrouve tour à tour prostrée, épuisée, insomniaque. Alors son père décide de la rapatrier précipitamment à Cotonou. A peine arrivée à Cotonou en novembre, elle se dresse contre C., sa «marâtre », et en criant « diable, diable » l'accuse d'être à la cause de sa maladie. On fait alors appel à un devin, puis à un prêtre exorciste. Tous deux affirment la culpabilité sorcière de Colette. Un mois plus tard, en décembre 1991, B. réunit, dans sa cour les deux familles, soit une vingtaine de personnes pour un conseil extraordinaire. La tension est extrême. Des paroles très dures seront prononcées de part et d'autre. C. demande sans succès d'être mise en présence de ses deux accusateurs. Alors, son oncle maternel apostrophe Bienvenu :

"Est-ce que C. a jamais fait quelque chose contre les enfants de R. ? C. a perdu un enfant à la naissance et a été malade durant un an. Elle n'a rien fait. Si c'est notre fille [qui s'avérait coupable], nous pourrons l'égorger. Si ce n'est pas elle alors il y aura un grand combat. Tu nous dis qu'il y a quelque chose de fétiche chez toi. On a sorti un bois avec fétiche de ta chambre. Mais c'est à toi ou à ta fille [E.] que l'on a fait quelque chose? Tu n'agis pas comme un homme, tu es très faible. Qui a trouvé le fétiche? C'est le fils de la soeur de R. B., là où tu vas c'est loin. Mais ma fille [ma nièce] a des enfants avec toi et on ne peut rien. Sinon tu veux créer une grande histoire de famille [conflit]. (...) Je suis chez toi mais tu ne peux rien me faire [pour t’avoir dit la vérité] ».

Désormais, E. n'est plus seule à accuser sa «marâtre »C. Son mari semble avoir désormais choisi son camp. Faute d'avoir été décisive, une deuxième réunion est organisée en janvier 1992 à Porto-Novo, dans la famille de B. Sans plus de succès. Toutefois, une fois la famille de $C$. repartie, B. prend la décision de chasser sa petite femme de la cour avec l'aide de sa famille. Le lendemain, à Cotonou, C., devant de sa chambre se saisit d'une hache et se dresse menaçante : "Je ne suis pas mariée avec vous. C'est la police qui me 
fera sortir. Si tu viens, je te tue et je me tue ». Fin provisoire apparente de l'épisode, après une autre bouffée de violence, les choses semblent rentrer dans l'ordre. Toutefois, deux mois plus tard, en mars 1992, à son retour du marché D., elle trouve la porte de sa chambre fermée à clé. Son époux avait changé le verrou pour lui en interdire l'accès. Elle ne quittera pas la cour pour autant et dormira dans la chambre de son plus jeune fils José Tous ses autres enfants fréquentaient [l'école] en dehors de Cotonou (un à Pobè, deux à Togoville, une à Porto-Novo). Entre mai et juin 1992, s'ensuivront trois nouvelles tentatives «mystiques» à l'initiative de Bienvenu pour la chasser de la maison conjugale. La première met en scène cinq féticheurs vodoun [de la religion vodu]. Durant deux semaines, ils multiplieront les cérémonies, après avoir enterré des fétiches protecteurs (glo*) aux quatre coins de la maison et au portail de la cour. La deuxième tentative est le fait d'un groupe de Christianistes Célestes, qui ont dressé des bougies dans la maison et allumé une lanterne. Sans plus de succès. La dernière tentative voit deux féticheurs répandre de la poudre anti-sorcière (nifi $i^{*}$ ) sur la porte de chambre de Colette. Echec à nouveau. C'est, en fin de compte, un huissier qui l'expulsera le 8 juillet 1992. "Réfugiée » dans le carré [concession] de sa mère et de son frère dans le quartier Aïdjedo, la situation de $C$. est désormais très précaire. Elle n'a ni logement, ni économies et ne percevra, bien entendu, jamais aucune compensation matérielle de son mari. Peu après cette journée, sa mère a un accident cardio-vasculaire et elle restera paralysée jusqu'à son décès en novembre 1996. Elle-même est durement éprouvée par la maladie (cancer du sein).

En août 1992, B. introduit une demande de divorce pour cause de sorcellerie auprès du Tribunal mais en dépit de ses relations privilégiées avec certains magistrats, la plainte ne sera pas jugée recevable, empêchant ainsi jusqu'en mars 2001 le mariage religieux entre $B$. et de R. Approché à plusieurs reprises, l'archevêque de Cotonou (Mgr de Souza) refusera de les marier. Et il interviendra pour faire échouer leurs tentatives réitérées de mariage religieux dans quatre paroisses différentes (une à Porto-Novo et trois à Cotonou) au cours de l'année 1999. Et ce n'est qu'à son décès en novembre 1999, que la cérémonie aura enfin lieu à la paroisse Sainte Cécile de Cotonou. «Tout a été dit, tout a été fait » conclut Colette. Elle n'a pas été la seule touchée par ce drame, chacun de ses enfants est resté personnellement marqué par cette violence qui s'est traduite par une réduction drastique du soutien paternel.

Loin d'être exceptionnel - les unions polygamiques y sont plus fréquemment prédisposées - ce témoignage n'illustre que mieux la réalité de la continuité et de la banalisation sociale de la violence, ce qui nous semble essentielle pour comprendre son développement au sein de l'arène familiale.

\section{La violence en partage}

Insister sur cette continuité sociale de la violence amène à considérer à la fois les liaisons entre champs sociaux différenciés, les jeux d'acteurs - mêlant histoires de vie, disparités socio-économiques et processus d'individuation - et l'environnement socio-politique immédiat qui concourent à la réplication temporelle et la diffusion spatiale de la violence. Toute la difficulté consiste à retrouver, derrière la diversité des situations vécues, une logique sociale qui permettre de rendre compte des processus en œuvre.

Il ne saurait exister de banalisation de la violence sans la multiplication au quotidien des micro-événements dans les diverses formes d'expérience et de situations sociales. Dans un groupe familial élargi à plusieurs dizaines de personnes, la violence verbale ou violence physique peut se cristalliser à partir d'incidents infimes mais « signifiants » pour la société qui les produit : ce peut être une sauce mal préparée, une consommation excessive de bois ou d'eau, une salutation mal rendue ou un remarque mal perçue... Les questions d'argent, qu'il s'agisse de dette éludée, de promesse non tenue, de remboursement reporté, servent très souvent de déclencheur. Dans l'ancienne cour de $\mathrm{C}$. à Cotonou, les «palabres en argent » 
sont ainsi fréquents. En 1998, G. au chômage annonce à son épouse M.-L., secrétaire, que son frère aîné, pharmacien de son état, souhaite revendre une buvette dont il est propriétaire à Porto-Novo. L'épouse lui remet alors 200.000 francs CFA. Plusieurs mois s'écoulent. MarieLouise interroge un jour son mari sur l'avancement de la transaction. Furieux, celui-ci réplique par des coups et jette l'ensemble des effets personnels de sa femme dans la rue : il avait dépensé, durant l'intervalle, tout l'argent que celle-ci lui avait confié.

D'abord circonscrite, la tension augmente généralement en fonction du nombre croissant des commentaires et des interventions croisées jusqu'à devenir générale. Parfois, au contraire, la violence diffuse se lit « en creux» : elle se déduit des attitudes et postures des uns et des autres, de leurs confidences et sous-entendus, à charge pour l'observateur de les décrypter. Dans la grande cour urbaine de Cotonou, personne ne laisse sa porte ouverte. Certains se plaignent d'un emprunt vestimentaire sans demande préalable, d'autres de la disparition d'un livre scolaire. B., le chef de famille, lui-même verrouille et déver-rouille systématiquement chaque porte sur son passage. Et si on s'apostrophe rarement d'un balcon à l'autre au sein de cette cour «à étages", les salutations que l'on se rend avec retard, comme si elles étaient extorquées, en disent long sur un long passé de dissensions et de ressentiment réciproques accumulés. En fin de compte, les tensions font partie de l'ordinaire le plus quotidien au même titre que l'incertitude et l'insécurité inhérentes aux conditions d'existence.

Nous avons déjà rappelé l'importance du contrôle social exercé par le chef de famille ou par la communauté dans l'émergence et la gestion (ou le laisser-faire) de la violence domestique, qu'elle soit villageoise ou familiale. Dans les deux cas présentés, en revanche, l'aggravation de la violence ordinaire est nourrie par l'épuisement relatif des figures classiques de régulation et la faiblesse des recours ${ }^{14}$. Vers qui peut-on se tourner dès lors que les aînés, les « grands », tous ceux qui sont dominants, entretiennent les meilleures relations possibles avec les recours éventuels? En milieu rural, la dérégulation s'exerce de manière encore plus sélective sur les personnes les plus vulnérables : femmes veuves, divorcées ou répudiées sans enfants. Dans les petites villes provinciales, le spectre social a tendance à s'élargir en liaison avec les processus d'individuation socio-économique. En revanche, en ville, ce phénomène est plus ancien et plus fréquent. On ne compte plus les chefs de ménages absentéistes ou fragilisés économiquement quand les choix familiaux n'imposent pas des placements et des regroupements qui donnent naissance à des unités résidentielles aux structures de parenté très complexes, sources de conflits accrus.

Dans la petite ville de Mani (est du Burkina Faso), un relatif discrédit pèse sur tel chef de cour, figure emblématique du pouvoir familial, pour ne pas avoir limité les agissements coupables des frères du défunt vis-à-vis de P., une femme veuve, après 1991. Dans cette vaste cour polygamique il n'y a plus de régulation efficace et centralisée des conflits. Chacun gère son ménage dans une autonomie relative, prend des décisions économiques, se déplace. Cet éclatement en plusieurs centres de décision est symboliquement confirmé par l'extrême difficulté qu'a le chef de cour à mobiliser «ses gens ", à les réunir lors de nos différentes enquêtes. Certains chefs de ménages contactés à plusieurs reprises, ne daignent même pas faire acte de présence au cours de toute une semaine. Dans cette cour, on était, en outre, passé en peu de temps d'une gestion communautaire des greniers céréaliers permettant une mutualisation des risques de disette - à une gestion plus individualisée (à défaut d'être foncièrement individualiste) des ressources ce qui s'avérait nettement plus défavorable aux plus vulnérables. La survie de chaque ménage dépendait de plus en plus de sa propre force de travail, de son niveau d'épargne ou des aides susceptibles d'être mobilisées.

A Cotonou, c'est la démission du père, qui a laissé libre cours aux jalousies mortifères des enfants de la première femme épousée coutumièrement vis-à-vis des autres demi-frères et demi-sœurs. Comment au sein de l'unité polygamique résidentielle s'est instauré ce déséquilibre qui a conduit le chef de famille à vivre sous l'influence d'une fraction du ménage ?

14 Janin 1999, p. 194. 
Les menées du «clan » de la première épousée peuvent s'expliquer partiellement par un faisceau croisé de jalousies liées à la réussite scolaire et à l'aisance relative de la deuxième femme et de ses enfants. Tout cela a été combiné avec un véritable procès d'accusation en sorcellerie (ce qui n'a rien d'exceptionnel au Bénin) qui a trouvé un terreau fertile dans les craintes physiques et symboliques du chef de famille. Dès lors, les liens d'alliance et de filiation se sont progressivement distendus. Peu à peu, B. a revu à la baisse les modalités de prise en charge de ses enfants : sur l'argent de poche et l'habillement d'abord, l'écolage et la santé ensuite, et enfin «l'accès au plat ». Une fois, C. chassée de la cour en 1992, la situation des enfants restés dans la cour s'est dégradée. La rupture n'a jamais été réellement consommée avec leur père mais pauvreté, ressentiment et désarroi ont été désormais leur lot commun ${ }^{15}$. Après avoir été privé de courant électrique, deux d'entre eux ont été mis à la porte en 1997. En 2001, un seul résidait encore dans la « grande maison ». Le désengagement financier de $B$. est patent tandis qu'une forme de générosité oblative et festive, tournée vers l'extérieur (amis et personnes non apparentées), se manifeste plus régulièrement à l'occasion d'événements symboliques (réussite d'un examen, mariage, naissance, funérailles...).

De même, la très forte rémanence de la violence domestique joue également un rôle dans la continuité et la banalisation de la violence. Ce phénomène s'explique par la nature même des régulations (et a fortiori par leur absence) au sein de la famille - arbitrages stéréotypés, décisions arbitraires et inéquitables, recherche outrancière du consensus, dissimulation des preuves et refus de la vérité. Il tient aussi à l'importance excessive que les victimes et dominés accordent à la place assignée par la société et leur famille. Plus la soumission et l'acceptation sont fortes, plus la situation est douloureusement vécue mais en silence. De ce fait, la prise en charge psychoaffective des victimes paraît très difficile : elle ne peut être réalisée par une personne extérieure et, dans le même temps, la probabilité qu'un membre du même groupe la réalise est faible. Chaque victime a été en mesure de restituer avec une stupéfiante précision, d'ordre quasi-photographique, l'enchaînement des événements, les postures individuelles, les paroles prononcées. A Cotonou, C. se rappelle avec une intensité renouvelée les conditions de son expulsion le 8 juillet 1992 :

«Il avait plu. Tout le quartier était en pleurs, en cris. Tous les enfants étaient sortis. J'étais seule. Je n'ai pas pleuré. Je lui ai dit [à B.] : je ne mérite pas. Tout ce que tu as fait, tu as bien fait [j'endure cette souffrance que tu m'apportes comme une épreuve chrétienne]. Deux policiers pleuraient. (...) J'ai pleuré cinq jours et cinq nuits chez ma maman ».

A Mani (est du Burkina Faso), P. a dû rassembler ses affaires, sous le regard des autres membres de la cour ou de leurs enfants. Ici et là, les efforts du curé de la paroisse ou les arbitrages du chef du village se sont révélés inefficaces pour canaliser cette violence. $\mathrm{Si}$, pour Pourga, une maigre compensation matérielle a pu être trouvée (une nouvelle case, une nouvelle parcelle de terre), en revanche Colette n'a bénéficié d'aucune aide. La mémoire affective des événements douloureux est d'autant plus vive qu'aucune médiation collective équitable n'a eu lieu.

Si l'idée qu'il existerait une sorte de continuité socio-géographique de la violence - par le transfert que l'on opère individuellement d'un milieu à l'autre n'est pas nouvelle, celle de la réversibilité potentielle de la violence (la victime devenant agresseur), favorisant sa reproduction au sein de l'arène de confrontations et de négociations entre les membres de la famille élargie, semble avoir été beaucoup moins explorée.

Dans cette cascade d'accusations en sorcellerie, qui a fini par gangrener l'ensemble de la famille de C., un épisode particulier de transfert d'accusation en sorcellerie a pu être isolé. En décembre 1991, au début de la phase de crise familiale paroxystique, W., un des fils de la

15 A. Marie, «Avatars de la dette communautaire. Crise des solidarités, sorcellerie et procès d'individualisation (itinéraires abidjanais », p. 270, in : A. Marie (éd.), L'Afrique des individus, Paris, Karthala, Collection »Homes et sociétés », 1997, pp. 249-328. 
deuxième épouse $C$. se remémore. Il aurait été étranglé en rêve. Son père lui explique qu'il avait failli être «mangé » par un sorcier. Et lui conseille de se rendre dans la famille paternelle à Porto-Novo, pour une séance de prière avec des membres de l'Eglise du Christianisme Céleste, où il s'est trouvé hébergé chez un demi-frère, le fils aîné de R. (la $1^{\circ}$ épouse). Mais, à son retour à Cotonou il est accusé d'avoir enterré un fétiche agressif (hobo*), une tête de canard garnie de fils, dans la maison et ce, pour le compte de sa mère Colette, qui avait déjà elle-même été accusée. De victime, W. était donc devenu un coupable !

De fait, les formes les plus fréquemment observées de violence familiale (rejet et exclusion, oubli et indifférence, jalousie, menace et chantage) se maintiennent, cheminant silencieusement avant d'être dévoilées, pour rejaillir par à-coups ou sous forme d'aveu involontaire dans d'autres sphères de la vie quotidienne. C'est M., la dernière fille de C. qui raconte les conséquences matérielles et psychoaffectives de ce conflit familial à rebondissement. Plus que la séparation déjà ancienne de ses parents en 1992, c'est le fait d'être à la fois indésirable dans la cour de son père et difficilement prise en charge par une mère très démunie qui a déclenché cette crise. Elle a alors développé un rejet global des contraintes sociales (et de la domination que cela impliquait), sous la forme d'une agressivité verbale et d'une instabilité psychoaffective au cours des années 1998 et 1999. Interviennent alors une série de sorties nocturnes prolongées, forme indirecte de fugues, qui sont autant d'occasions de chercher une «protection » ou de nouvelles sources de revenus. Ce comportement exprimait alors un désarroi profond et réel tout en générant une violence de «basse intensité ».

Dans les sociétés de castes africaines (et elles sont nombreuses), la violence interindividuelle, nous l'avons rappelé, se nourrit d'abord des inégalités de statut et des différences d'âge. On aurait cependant tort de croire qu'un rapport de force moins déséquilibré, au sein d'une fratrie par exemple, minimise tensions et conflits. Bien au contraire, la proximité et l'égalité tendancielle rendent les arbitrages plus fragiles et plus aléatoires ${ }^{16}$ et réactivent la violence.

A Cotonou, Ch. (une des fils de C.) a prêté (emprunté) de l'argent à sœur aînée. Il n'a de cesse de minimiser cette créance, mettant en avant sa condition de chômeur par rapport à sa sœur qui serait garantie (sécurisée financièrement) par son mariage. La date d'un éventuel remboursement est naturellement repoussée. Après plusieurs discussions vives, il annonce même qu'il ne fera rien. Puis, très rapidement, il se retourne et s'adresse à son frère «même père, même mère » pour l'apostropher : «tu me dois ». Le ton monte, chacun s'efforçant de parler «plus haut », de garder un ascendant psychologique. Les invectives pleuvent. Chacun mobilise les arguments pour prendre une tierce personne à témoin et pour pointer du doigt l'inconduite notoire de l'autre. « Après ce que j'ai fait pour toi,... c'est moi qui ai payé le manger qui te profite... tu es faux...». La tension atteint un point extrême. Il importe à chacun de se mettre en scène pour convaincre : pointer un doigt menaçant, alterner mimiques offusquées, gestes vengeurs et poses défensives, tout ceci paraît dérisoire ou excessif. Et pourtant... Les efforts de chacun pour évacuer toute responsabilité est prodigieuse tout comme la violence dans la rhétorique visant à dénoncer le comportement de l'autre, violence d'autant plus ouverte qu'elle est entretenue par le refus martelé de concéder quoi que ce soit. D'ailleurs à y regarder de près, le jugement que l'on porte sur autrui est très souvent caricatural.

Chacun est donc, tour à tour, le « grand » et le « petit » d'un autre pour reprendre des termes à connotations sociales et psychologiques très fortes en Afrique de l'Ouest. Ce qui revient à dire, si on les analyse en terme de pouvoir établi, que chaque individu est, à la

\footnotetext{
16 «Cette hiérarchisation de la société n'est pas un acquis et reste plus ou moins bien acceptée. D’où cette tension permanente entre acceptation et refus, discours et pratique, qui mine les individus et peut conduire à s'opposer symboliquement ou concrètement à l'ordre social. A l'inégalité fondamentale des statuts et des conditions qui suppose une soumission sans faille s'oppose en permanence la revendication démocratique exacerbée par l'individualisme de la société masculine et, de plus en plus, la désespérance actuelle des jeunes » (Janin 1999, p. 180).
} 
fois, dominé et dominant, abuseur et abusé, «endetté insolvable » et « créancier floué ${ }^{17}$ selon les différentes relations à autrui qui tissent la vie quotidienne au sein des milieux sociaux de type communautaire. Il n'y a donc pas de position dominante unilatérale et irréversible ni de responsabilité établie une fois pour toutes dans la répartition et dans la diffusion de la violence.

La famille constitue donc un laboratoire social exemplaire pour lire les ruptures et repérer les tensions qui travaillent des sociétés de structure encore largement communautaire, confrontées à des changements rapides. Contre toute attente, les violences multiformes qui traversent les familles ne s'enracinent pas nécessairement dans des situations de violence civile ou politique extrême ni même dans la paupérisation économique, même si elles sont susceptibles de les aggraver ; elles renvoient plus fondamentalement à la nature même des rapports interpersonnels entre membres du groupe, faits de jalousie, de ressentiment mais également de convivialité, de détente et d'entraide mutuelle.

L'âpreté mordante des relations au sein du couple ou de la fratrie n'est donc pas permanente mais les compromis équitables négociés, les attentions tendres et désintéressées sont plus rares que les solidarités calculées et les dettes à répétition, exprimant un utilitarisme commode ou une forme de parasitisme social. Etant donné le caractère hiérarchique ou autoritaire des relations entre aînés et cadets, entre parents et enfants, la parole sert moins à apaiser, à réconforter qu'à produire de la contrainte, du contrôle et de la crainte. Penser à l'autre c'est le plus souvent se trouver pris dans des supputations plus ou moins soupçonneuses ou utilitaristes d'instrumentalisation implicites croisées. Dans le respect codifié comme dans l'ostentation parfois quelque peu méprisante, on cherchera souvent vainement des marques de sociabilité constructive pour la personne. De même, quel sens donner au terme de vérité, lorsque chacun brouille les pistes au nom d'une pluralité des normes bien commode ${ }^{18}$ ? Comment replacer la responsabilité au cœur de la relation à l'autre si la communauté ne la reconnaît pas, si les arbitrages qu'elle propose sont des arrangements, presque toujours sélectifs, temporaires et circonscrits ?

Si la violence constitue une sorte d'invariant comportemental au sein de la famille, c'est non seulement parce que celle-ci est perméable aux différentes formes de violence « importée » (de l'école, de la rue) mais aussi parce que les régulations proposées ne sont pas de nature à y apurer les conflits et jouent sur le registre de la confusion. Même lors des concertations familiales, il est très difficile de hiérarchiser les événements, de sérier les causalités et, a fortiori, de définir les responsabilités. Si l'on confronte les discours de chaque protagoniste on perçoit rapidement le hiatus existant entre vérité objective (ce qui s'est effectivement passé mais ne sera peut-être jamais dévoilé), vérité requise (ce que l'on cherche à découvrir et à faire connaître), vérité déclarée (ce que l'on apprend par la rumeur ou la confidence) et vérité officiellement proclamée (ce qui est annoncé publiquement après recherche apparente d'un consensus qui est, en réalité, imposé par des rapports de force).

Pierre JANIN. IRD- Ouagadougou

IEDES - Université de Paris I

\footnotetext{
17 A. Marie, «Y pas l'argent : l'endetté insolvable et le créancier floué, deux figures complémentaires de la pauvreté abidjanaise », Paris, Puf, Revue Tiers-Monde, 1995, XXXVVI, 142, 303-324.

18 J.-P. Chauveau et al., "La pluralité des normes et leurs dynamiques en Afrique », pp. 145-162, in : G. Winter (éd.), Inégalités et politiques publiques en Afrique, Paris, Karhala, 2001, 454 p.
} 